\title{
COEFICIENTES DE INTERFACE PARA TRANSFERÊNCIA DE CALOR E ÁGUA EM BIORREATORES DE FERMENTAÇÃO SÓLIDA
}

\author{
F. P. CASCIATORI ${ }^{1}$, A. BÜCK ${ }^{2}$, E. TSOTSAS ${ }^{2}$ e J. C. THOMÉO ${ }^{1}$ \\ ${ }^{1}$ Universidade Estadual Paulista, Departamento de Engenharia e Tecnologia de Alimentos \\ ${ }^{2}$ Otto-von-Guericke Universität, Thermal Process Engineering, NaWiTec \\ E-mail para contato: fernandacasciatori@yahoo.com.br
}

RESUMO - Dentre várias possíveis aplicações industriais, leitos empacotados podem ser empregados como biorreatores de fermentação em estado sólido (FES), processo biotecnológico no qual uma matriz sólida porosa é fermentada por fungos. Modelos de duas fases são apropriados para predizer a transferência de calor e de água nesses biorreatores, mas dependem do conhecimento dos coeficientes de transferência de calor $(\alpha)$ e de massa $(\beta)$ na interface gás-sólido e da razão de área superficial de troca por unidade de volume (At/V). Visando dar suporte a trabalhos de modelagem e simulação de biorreatores de FES, o objetivo do presente trabalho foi propor uma metodologia para cálculo de tais coeficientes com base em correlações de Nusselt $(\mathrm{Nu})$ e Sherwood (Sh) e empregar o conceito de volumes-finitos para calcular At/V. Para $\mathrm{Nu}$, a geometria da partícula e a direção do escoamento do fluido foram consideradas e correlações clássicas foram empregadas. Com base na analogia entre transferência de calor e de massa, Sh foi considerado numericamente igual a Nu. Para cálculo de At/V, a geometria das partículas do substrato foi considerada e o leito foi dividido em elementos finitos. Deste modo, os coeficientes de interface podem ser calculados para biorreatores empacotados com diferentes tipos partículas, para leitos de diferentes geometrias e para várias condições operacionais.

\section{INTRODUÇÃO}

Leitos empacotados são extensivamente usados em importantes operações unitárias nas indústrias químicas, de alimentos e de processos em geral. Alguns exemplos de aplicação incluem seus usos como separadores, absorvedores, secadores, filtros, trocadores de calor e reatores químicos e bioquímicos (Thoméo et al., 2004). Os biorreatores de fermentação em estado sólido (FES) são um caso particular de leitos empacotados que estão em destaque na literatura. A FES pode ser definida como o crescimento de microrganismos sobre partículas sólidas úmidas, em situações nas quais o espaço entre as partículas contêm uma fase gasosa contínua e a água está ou impregnada nas partículas ou forma um fino filme de água sobre elas. Entretanto, o conteúdo de água da matriz porosa deve ser suficientemente alto para assegurar adequadas condições de crescimento ao metabolismo microbiano (Mitchell et al., 2006). 


\section{9 a 22 de outubro de 2014 \\ Florianópolis/SC}

Os biorreatores de leito empacotado são os mais comumente aplicados na FES devido ao seu simples projeto, reduzido custo e baixas exigências de manutenção, sendo, por isso, o foco deste trabalho. Ademais, a ausência de movimentação das partículas torna estes biorreatores ideais para microrganismos sensíveis às tensões de cisalhamento, como é o caso de alguns fungos filamentosos, que podem ter suas hifas rompidas com a movimentação do leito, o que prejudicaria o desenvolvimento do microrganismo.

Apesar de seu potencial de aplicação, biorreatores de FES são muito raramente operados como leitos empacotados em larga escala ao longo de todo o período de cultivo. Um dos principais problemas na operação dos biorreatores de leito empacotado é a deficiente remoção do calor gerado metabolicamente, em decorrência de fatores associados tanto ao tipo de substrato quanto às condições de operação. Os resíduos agroindustriais comumente empregados como substratos, devido ao fato de serem materiais orgânicos, possuem condutividades térmicas muito baixas, o que dificulta a dispersão do calor metabólico por condução. Ademais, para operar o biorreator como leito fixo, as vazões de ar através do leito não podem ser muito elevadas, para evitar a secagem excessiva do substrato, por consequência da remoção de água pelo ar, em decorrência do não equilíbrio termodinâmico entre o ar de percolação e o substrato sólido. Deste modo, a remoção de calor por convecção também é limitada, o que resulta em gradientes axiais de temperatura e consequentes gradientes de umidade.

A modelagem de biorreatores de FES e a simulação desses sistemas reacionais em leitos empacotados mostram-se como importantes ferramentas para o desenvolvimento desses biorreatores, uma vez que podem fornecer informações de prováveis pontos quentes e secos no interior do leito e alternativas operacionais de como evitar estes problemas (Thoméo et al., 2004). Assim, modelos que descrevam os mecanismos de transferência de calor e de água em biorreatores de FES podem fornecer bons indicativos do que ocorrerá no sistema antes de prosseguir diretamente aos experimentos.

O conceito de modelagem em FES é a busca por expressões matemáticas que representem o sistema reacional, de forma que os modelos possam ser usados como ferramentas no dimensionamento de biorreatores de larga escala. Essas expressões têm o objetivo de descrever como o desempenho dos biorreatores é afetado pelas variáveis operacionais que podem ser manipuladas na tentativa de manter o processo sob controle. Por exemplo, um modelo matemático pode predizer como a taxa de escoamento do ar, sua umidade relativa e temperatura irão afetar a temperatura e o conteúdo de umidade do substrato e também como essas variáveis ambientais irão afetar o crescimento microbiano e a formação de produtos (Mitchell et al., 2003).

Saucedo-Castaneda et al. (1990) foram um dos pioneiros na proposição de um modelo de transferência de calor na FES em leitos empacotados, tendo aplicado um balanço geral de transferência de calor sem considerar dispersões axial e radial. Sangsurasak e Mitchell (1995 a, b) melhoraram este modelo original, considerando a transferência de calor bidimensional em um modelo adequado a várias geometrias e condições operacionais. Mais tarde, Sangsurasak e Mitchell (1998) estenderam seu próprio modelo anterior incluindo um termo para descrever a evaporação de água da matriz porosa, que contribui significativamente para a remoção de calor global, apesar de que este fenômeno resulta na secagem do leito (Gutierrez-Rojas et al., 1996). Todos esses modelos são pseudo-homogêneos, isto é, consideram que, em um elemento de volume representativo do sistema, 


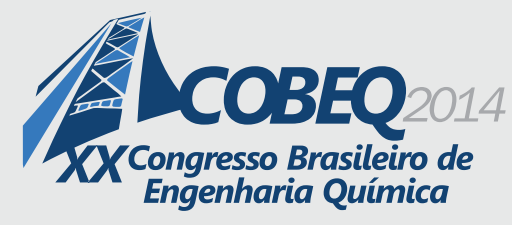

19 a 22 de outubro de 2014

Florianópolis/SC

as temperaturas e as atividades de água são iguais para ambas as fases sólida e fluida. O modelo de Sangsurasak e Mitchell (1998) é muito similar aos modelos pseudo-homogêneos de modelagem da transferência de calor em reatores químicos resfriados pela parede, uma vez que o crescimento fúngico pode ser tratado no modelo como uma reação exotérmica (Vortmeyer e Haidegger, 1991). Entretanto, a principal deficiência desses modelos pseudo-homogêneos é que eles predizem a transferência de água do sólido para o ar, mas não são adequados para predizer a secagem do leito. Somente modelos heterogêneos com pelo menos duas fases são capazes de representar bem o fenômeno de secagem do leito ao longo da fermentação em decorrência da percolação de ar.

Von Meien e Mitchell (2002) foram os primeiros a sugerir um modelo de duas fases para a predição de gradientes de temperatura e umidade em um biorreator de FES agitado intermitentemente com aeração forcada, tendo modelado apenas os estágios em que o biorreator operava como leito empacotado, entre os sucessivos estágios de agitação, usando a mesma aproximação aplicada para a secagem de grãos em leitos fixos. Embora a implementação dos modelos de duas fases requeira as informações fluido-partícula, esses modelos são mais realistas e são os únicos capazes de representar adequadamente a transferência de água, e consequentemente de calor evaporativo, da fase sólida para a gasosa. O sistema foi dividido em uma fase sólida, contendo água e sólidos secos, e uma fase fluida, incluindo vapor d'água e ar seco. O modelo foi então constituído por balanços de umidade e de calor nas fases sólida e gás, Equações 1 e 2 e Equações 3 e 4, respectivamente:

$$
\begin{aligned}
& \frac{\partial(\mathrm{SX})}{\partial \mathrm{t}}=-\beta \mathrm{a}\left(\mathrm{X}-\mathrm{X}^{*}\right)+\mathrm{R}_{\mathrm{w}}\left(\mathrm{S} \frac{\partial \mathrm{X}}{\partial \mathrm{t}}+\mathrm{X} \frac{\partial \mathrm{S}}{\partial \mathrm{t}}\right) \\
& \varepsilon \rho_{\mathrm{g}} \frac{\partial \mathrm{Y}}{\partial \mathrm{t}}+\rho_{\mathrm{g}} \mathrm{v}_{0} \frac{\partial \mathrm{Y}}{\partial \mathrm{z}}=\beta \mathrm{a}\left(\mathrm{X}-\mathrm{X}^{*}\right) \\
& \mathrm{S}\left(\mathrm{Cp}_{\mathrm{s}}+\mathrm{XCp_{ \textrm {w } }}\right) \frac{\partial \mathrm{T}_{\mathrm{s}}}{\partial \mathrm{t}}=\mathrm{ha}\left(\mathrm{T}_{\mathrm{g}}-\mathrm{T}_{\mathrm{s}}\right)-\lambda \beta \mathrm{a}\left(\mathrm{X}-\mathrm{X}^{*}\right)+\mathrm{R}_{\mathrm{Q}}\left(\mathrm{S} \frac{\partial \mathrm{X}}{\partial \mathrm{t}}+\mathrm{X} \frac{\partial \mathrm{S}}{\partial \mathrm{t}}\right) \\
& \varepsilon \rho_{\mathrm{g}}\left(\mathrm{Cp}_{\mathrm{g}}+\mathrm{YCp}_{\mathrm{v}}\right) \frac{\partial \mathrm{T}_{\mathrm{g}}}{\partial \mathrm{t}}+\left(\mathrm{Cp}_{\mathrm{g}}+\mathrm{YCp_{ \textrm {v } }}\right) \rho_{\mathrm{g}} \mathrm{v}_{0} \frac{\partial \mathrm{T}_{\mathrm{g}}}{\partial \mathrm{z}}=-\mathrm{ha}\left(\mathrm{T}_{\mathrm{g}}-\mathrm{T}_{\mathrm{s}}\right)
\end{aligned}
$$

onde $\mathrm{S}$ é a concentração volumétrica de sólidos secos no leito $\left(\mathrm{kg}\right.$-sólidos $\left./ \mathrm{m}^{3}\right) ; \beta$ a é o coeficiente de transferência de água na interface $\left(\mathrm{kg}\right.$-água $\left./ \mathrm{s} / \mathrm{m}^{3}\right)$; b é a fração de biomassa nos sólidos secos $(\mathrm{kg}$ biomassa/kg-sólidos); $\mathrm{X}$ é a umidade da fase sólida e $\mathrm{X}^{*}$ seu valor no equilíbrio (kg-água/kg-sólidos); $\mathrm{R}_{\mathrm{W}}$ é o coeficiente estequiométrico para produção de água durante o crescimento fúngico (kgágua/kg-biomassa); Y é a umidade do ar (kg-água/kg-ar); $\mathrm{T}_{\mathrm{s}}$ e $\mathrm{T}_{\mathrm{g}}$ são as temperaturas das fases sólida e gasosa $\left({ }^{\circ} \mathrm{C}\right)$, respectivamente; ha é o coeficiente de transferência de calor na interface sólido-fluido $\left(\mathrm{W} / \mathrm{m}^{3} / \mathrm{K}\right) ; \mathrm{Cp}_{\mathrm{s}}$ é o calor específico dos sólidos secos $(\mathrm{J} / \mathrm{kg}$-sólidos $/ \mathrm{K}) ; \mathrm{Cp}_{\mathrm{w}}$ é o calor específico da água líquida $\left(\mathrm{J} / \mathrm{kg}\right.$-água/K); $\mathrm{Cp}_{\mathrm{g}}$ é o calor específico do ar seco $(\mathrm{J} / \mathrm{kg}-\mathrm{ar} / \mathrm{K}) ; \mathrm{Cp}_{\mathrm{v}}$ é o calor específico do vapor d'água $(\mathrm{J} / \mathrm{kg}$-vapor/K). A cinética de crescimento fúngico foi dada pelo modelo logístico e a variação do conteúdo de sólidos totais foi obtida por um balanço de massa aplicado aos sólidos secos. 


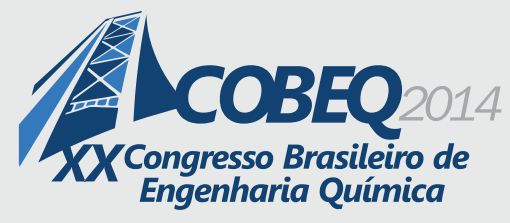

19 a 22 de outubro de 2014
Florianópolis/SC

Nas Equações 1 e 2, os termos contendo o parâmetro ßa representam a transferência de água na interface sólido-gás, enquanto nas Equações 3 e 4 os termos contendo o parâmetro ha representam a transferência de calor na interface. No entanto, apesar da complexidade do modelo de Von Meien e Mitchell (2002), os coeficientes de transferência na interface sólido-gás $\beta$ a e ha, que regem os termos de transferência de massa e de calor entre as fases, respectivamente, foram obtidos da literatura de secagem de grãos em leito fixo e não necessariamente correspondem ao sistema de FES. Schutyser $e t$ al. (2004) também empregaram um modelo de duas fases para prever perfis térmicos e de umidade durante os estágios da FES em que o substrato permanecia estático em seu biorreator de tambor rotativo, e também não deixaram clara a obtenção dos coeficientes de transferência na interface.

Neste contexto, o objetivo do presente trabalho foi propor uma metodologia para cálculo desses coeficientes de transferência de calor e de água na interface gás-sólido nos biorreatores de FES em leito fixo com base em correlações clássicas de Nusselt $(\mathrm{Nu})$ e Sherwood $(\mathrm{Sh})$, respectivamente, levando em consideração a geometria das partículas do substrato e as condições operacionais do sistema fermentativo, bem como empregar o conceito de volumes-finitos para calcular a razão área de troca na interface por unidade de volume do reator (At/V) para escalonamento desses coeficientes, visando a dar suporte a trabalhos de simulação de biorreatores de FES em leito fixo empregando modelos heterogêneos para previsão de perfis térmicos e de umidade ao longo do processo.

\section{METODOLOGIA DE CÁLCULO}

\subsection{Caso para estudo}

O sistema fermentativo escolhido como estudo de caso para o cálculo dos coeficientes de interface e da relação área de troca por unidade de volume foi um sistema de produção de enzimas celulolíticas, fundamentais na etapa de hidrólise enzimática da biomassa vegetal na cadeia de produção do etanol de segunda geração, sendo tais enzimas responsáveis pela degradação da celulose em açúcares fermentescíveis. No entanto, o procedimento de cálculo pode ser aplicado a diferentes sistemas, uma vez que as equações foram desenvolvidas em termos de variáveis que podem ser substituídas pelos valores apropriados para cada sistema fermentativo.

O sistema para estudo de caso consistiu de um biorreator de leito empacotado no qual o fungo termofílico recentemente isolado Myceliophthora thermophila I-1D3b foi cultivado em bagaço de cana e farelo de trigo $(7: 3 \mathrm{~m} / \mathrm{m})$, no qual altos níveis de produção de celulases foi atingido por Zanelato et al. (2012) e Casciatori et al. (2013). O biorreator de leito empacotado era cilíndrico e tinha $76.2 \mathrm{~mm}$ de diâmetro e $1 \mathrm{~m}$ de comprimento. A velocidade superficial do ar percolante foi de 0.015 $\mathrm{m} / \mathrm{s}$. A porosidade da matriz sólida foi assumida constante e igual a 0.75 (Laurentino, 2007). O biorreator operava como leito empacotado estático durante todo o tempo de fermentação.

\subsection{Cálculo dos Coeficientes de Transferência de Calor e de Massa}

A proposta do presente trabalho foi calcular os coeficientes de transferência de calor e de massa na interface gás-sólido a partir de correlações clássicas encontradas na literatura para Nusselt $(\mathrm{Nu})$ e Sherwood (Sh), respectivamente para calor e massa. Para tanto, as partículas no leito foram assumidas 
como cilindros com diâmetro de partícula $\mathrm{d}_{\text {part }}=0.46 \mathrm{~mm}$ e comprimento de partícula $\mathrm{L}_{\text {part }}=15 \mathrm{~mm}$, $70 \%$ das mesmas tendo ar em fluxo cruzado e $30 \%$ em fluxo paralelo. Assim, dois diferentes números de Nusselt foram calculados por correlações apropriadas para cilindros em fluxo cruzado $\left(\mathrm{Nu}_{\mathrm{cf}}\right)$ ou em fluxo paralelo $\left(\mathrm{Nu}_{\mathrm{pf}}\right)$, então um número de Nusselt ponderado $\left(\mathrm{Nu}_{\mathrm{w}}\right)$ foi obtido.

Uma vez que os biorreatores de FES são percolados por ar e considerando válida a analogia entre transferência de calor e de massa, os números de Nusselt $\left(\mathrm{Nu}_{\mathrm{w}}\right)$ e de Sherwood $\left(\mathrm{Sh}_{\mathrm{w}}\right)$ ponderados foram numericamente igualados, já que o número de Lewis para o sistema ar-água ( $\mathrm{Le}=$ $\mathrm{Nu} / \mathrm{Sh}$ ) é aproximadamente igual a 1 . Deste modo, foi possível calcular simultaneamente os coeficientes de transferência de calor (a partir de $\mathrm{Nu}_{\mathrm{w}}$ ) e de massa (a partir de $\mathrm{Sh}_{\mathrm{w}}$ ) na interface.

Para ar em fluxo cruzado com as fibras cilíndricas, a correlação de Churchill e Bernstein (1977) foi empregada:

$$
\mathrm{Nu}_{\mathrm{cf}}=0.3+\frac{0.62 \operatorname{Re}_{\mathrm{p}}^{1 / 2} \operatorname{Pr}^{1 / 3}}{\left[1+(0.4 / \mathrm{Pr})^{2 / 3}\right]^{1 / 4}}\left[1+\left(\frac{\operatorname{Re}_{\mathrm{p}}}{282000}\right)^{5 / 8}\right]^{4 / 5}
$$

onde $\mathrm{Nu}_{\mathrm{cf}}$ é o número de Nusselt para os $70 \%$ de fibras com ar em fluxo cruzado.

Para ar em fluxo paralelo às fibras, o cilindro infinito foi aproximado para uma placa plana, e então a seguinte correlação clássica para placas foi empregada (Lienhard IV e Lienhard V, 2000):

$\mathrm{Nu}_{\mathrm{pf}}=0.664 \operatorname{Re}_{\mathrm{pf}}^{0.5} \operatorname{Pr}^{1 / 3}$

onde $\mathrm{Nu}_{\mathrm{pf}}$ é o número de Nusselt para os $30 \%$ de fibras com ar em fluxo paralelo e (para placa plana).

O número de Nusselt ponderado $\left(\mathrm{Nu}_{\mathrm{w}}\right)$ foi então dado por: $\mathrm{Nu}_{\mathrm{w}}=0.7 \mathrm{Nu}_{\mathrm{cf}}+0.3 \mathrm{Nu}_{\mathrm{pf}}$

Com base na analogia entre transferência de calor e de massa: $\quad \mathrm{Nu}_{\mathrm{w}}=\mathrm{Sh}_{\mathrm{w}}$

\subsection{Cálculo da Razão Área de Troca por Unidade de Volume}

Ambos os coeficientes de transferência de calor (h) e de massa $(\beta)$ na interface gás-sólido devem ser escalonados pela razão entre a área total de troca e o volume total do biorreator (At/V, $\mathrm{m}^{2} / \mathrm{m}^{3}$ ), a fim de englobar a transferência na interface em todo o biorreator. Para cálculo dessa razão, foram feitas as seguintes considerações:

- Uma fibra de bagaço de cana foi assumida como um cilindro com diâmetro e comprimento conhecidos, de modo que o volume de uma única fibra ( $\left.\mathrm{V}_{\text {uma-fibra }}\right)$ pôde ser calculado como:

$\mathrm{V}_{\text {uma-fibra }}=\pi \frac{\mathrm{D}_{\text {fibra }}^{2}}{4} \mathrm{~L}_{\text {fibra }}$ 
- A porosidade média do leito $\left(\varepsilon_{0}\right)$ foi obtida para uma larga faixa de conteúdos de umidade (Laurentino, 2007). Considerando um elemento de volume do biorreator como uma fatia de seção transversal $\mathrm{A}_{\text {app }}$ e comprimento dz, o volume total de sólidos ( $\mathrm{V}_{\text {fibras-elemento }}$ é dado por:

$\mathrm{V}_{\text {fibras-elemento }}=\left(1-\varepsilon_{0}\right) \mathrm{dV}$

- O número de fibras em um elemento de volume ( $\left.\mathrm{N}_{\text {fibras-elemento }}\right)$ foi então computado por:

$\mathrm{N}_{\text {fibras-elemento }}=\frac{\mathrm{V}_{\text {fibras-elemento }}}{\mathrm{V}_{\text {uma-fibra }}}$

- A área de uma única fibra $\left(\mathrm{A}_{\text {uma-fibra }}\right)$ é dada por: $\mathrm{A}_{\text {uma-fibra }}=\pi\left(\mathrm{D}_{\mathrm{fibra}} \mathrm{L}_{\mathrm{fibra}}\right)$

- A área total das fibras em um elemento de volume $\left(\mathrm{A}_{\text {fibras-elemento }}\right)$ é então calculada como:

$A_{\text {fibras-elemento }}=N_{\text {fibras-elemento }} A_{\text {uma-fibra }}$

$A_{\text {fibras-elemento }}=\frac{V_{\text {fibras-elemento }}}{V_{\text {uma-fibra }}} A_{\text {uma-fibra }}=\frac{\left(1-\varepsilon_{0}\right) d V}{V_{\text {uma-fibra }}} A_{\text {uma-fibra }}=\left(1-\varepsilon_{0}\right) A_{\text {app }} \frac{A_{\text {uma-fibra }}}{V_{\text {uma-fibra }}} d z$

$\mathrm{A}_{\text {fibras-elemento }}=\left(1-\varepsilon_{0}\right) \mathrm{A}_{\text {app }} \frac{\pi\left(\mathrm{D}_{\text {fibra }} \mathrm{L}_{\text {fibra }}\right)}{\frac{\pi}{4}\left(\mathrm{D}_{\text {fibra }}^{2} \mathrm{~L}_{\text {fibra }}\right)} \mathrm{dz}=\left(1-\varepsilon_{0}\right) \mathrm{A}_{\text {app }} \frac{4}{\mathrm{D}_{\text {fibra }}} \mathrm{dz}$

- Finalmente, para um elemento de volume, a razão At/V foi calculada por:

$\mathrm{a}=\mathrm{At} / \mathrm{V}=\frac{\mathrm{A}_{\text {fibras-elemento }}}{\mathrm{V}_{\text {elemento }}}=\frac{\left(1-\varepsilon_{0}\right) \mathrm{A}_{\text {app }} \frac{4}{D_{\text {fibra }}} \mathrm{dz}}{\mathrm{A}_{\text {app }} \mathrm{dz}}=\left(1-\varepsilon_{0}\right) \frac{4}{\mathrm{D}_{\text {fibra }}}$

$\mathrm{O}$ valor de $\mathrm{a}=\mathrm{At} / \mathrm{V}$ para um elemento de volume é constante para o biorreator como um todo. Assim, embora os coeficientes h e $\beta$ tenham sido calculados para uma partícula, ao serem multiplicados por At/V, os coeficientes ha e $\beta$ a estão escalonados.

\subsection{Extensão do Cálculo para Partículas e Leitos de Diferentes Geometrias e para Diferentes Condições Operacionais}

A metodologia de cálculo desenvolvida, embora tenha sido empregada para um estudo de caso específico, é passível de ser aplicada para vários outros biorreatores de FES em leito fixo.

Primeiramente, com relação aos coeficientes de transferência de calor (h) e massa $(\beta)$ na interface gás-sólido, a escolha da correlação apropriada para cálculo de Nusselt leva em conta a 


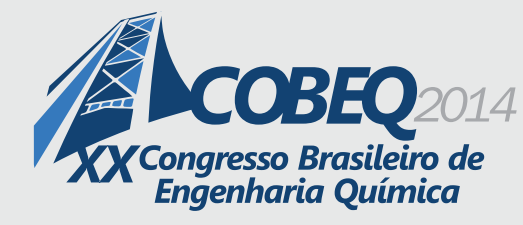

19 a 22 de outubro de 2014
Florianópolis/SC

geometria das partículas do substrato e as condições operacionais, particularmente a direção do escoamento e a velocidade superficial do fluido, englobadas no número de Reynolds (Re). Se, por exemplo, o substrato da fermentação é composto majoritariamente por partículas que podem ser consideradas aproximadamente esféricas, tais como grãos de soja, grânulos de amido ou ainda beads poliméricas embebidas com substrato, a correlação de Whitaker (1972) pode ser usada em lugar das Equações 5 a 7 para cálculo de $\mathrm{Nu}$ para ar escoando ao redor de partículas esféricas, inclusive para baixas faixas de Re, típicas de biorreatores de FES. É necessário apenas que a geometria e as dimensões das partículas do substrato sejam conhecidas, bem como a direção, velocidade superficial e as propriedades físicas do ar na temperatura do processo.

Com relação à razão At/V, o desenvolvimento das Equações 9 a 16 também pode ser facilmente adaptado para partículas e para leitos de diferentes geometrias e dimensões, desde que as fórmulas geométricas apropriadas sejam aplicadas para calcular o volume da partícula e o volume do elemento ou fatia do biorreator. Neste caso, além de conhecer a geometria e as dimensões das partículas e do leito, também é necessário que a porosidade média do leito $\left(\varepsilon_{0}\right)$ seja conhecida. Não há restrições para cálculo de At/V, tendo em vista que esta propriedade não depende das condições operacionais empregadas.

\section{CONSIDERAÇÕES FINAIS}

No presente trabalho, se apresentou uma metodologia para cálculo dos coeficientes de transferência de calor e de umidade na interface gás-sólido em um biorreator de FES em leito empacotado com base em correlações de Nusselt (Nu) e Sherwood (Sh), bem como se empregou o conceito de volumes-finitos para cálculo da razão área de troca por unidade de volume do biorreator.

Como a escolha das correlações para cálculo dos adimensionais de calor e de massa e o desenvolvimento das equações para cálculo da razão área de troca por volume levaram em conta a geometria da partícula, a direção do escoamento do fluido percolante e a geometria do leito, o procedimento de cálculo aqui desenvolvido possibilita calcular tais coeficientes de interface para biorreatores empacotados com diferentes tipos partículas, para leitos de diferentes geometrias e para várias condições operacionais.

\section{REFERÊNCIAS}

CASCIATORI, F. P.; CASCIATORI, P. A.; THOMÉO, J. C. Cellulase production in packed bed bioreactor by solid-state fermentation. In: European Biomass Conference and Exhibition Proceedings, p. 1539-1546, 2013.

CHURCHILL, S. W.; BERNSTEIN, M. A Correlation Equation for Forced Convection from Gases and Liquids to a Circular Cylinder in Cross Flow. J. Heat Transfer, Trans. ASME, v. 94, p. 300-306, 1977.

GUTIERREZ-ROJAS, M.; HOSN, S.A.A.; AURIA, R.; REVAH, S.; FAVELA-TORRES, E. Heat transfer in citric acid production by solid state fermentation. Process Biochem. v. 31, p. 363-369, 1996. 
LAURENTINO, C. L. Transferência de calor em leitos empacotados para aplicação em biorreatores de fermentação em estado sólido. 2007. 105f. (Dissertação de Mestrado). PPGECA/UNESP, São José do Rio Preto, Brasil, 2007.

LIENHARD IV, J. H.; LIENHARD V, J. H. A heat transfer textbook. Cambridge: J. H. Lienhard V, 2000.

MITCHELL, D. A.; KRIEGER, N.; BEROVIC, M. Solid-state fermentation bioreactors: fundamentals, design and operation. Berlin: Springer-Verlag, 2006.

MITCHELL, D. A.; VON MEIEN, O. F.; KRIEGER, N. Recent developments in modeling of solid-state fermentation: heat and mass transfer in bioreactors. Biochem. Eng. J., v. 13, p. 137-147, 2003.

SANGSURASAK, P.; MITCHELL, D. A. The investigation of transient multidimensional heat transfer in solid state fermentation. Chem. Eng. J., v. 60, p. 199-204, 1995 a.

SANGSURASAK, P.; MITCHELL, D. A. Incorporation of death kinetics into a 2-D dynamic heat transfer model for solid state fermentation. J. Chem. Technol. Biotechnol., v. 64, p. 253-260, $1995 b$.

SANGSURASAK, P.; MITCHELL, D.A. Validation of a model describing two-dimensional heat transfer during solid-state fermentation in packed bed bioreactors. Biotechnol. Bioeng., v. 60, p. 739-749, 1998.

SAUCEDO-CASTAÑEDA, G.; GUTIERREZ-ROJAS, M.; BACQUET, G.; RAIMBAULT, M.; VINIEGRA-GONZÁLEZ, G. Heat transfer simulation in solid substrate fermentation. Biotechnol. Bioeng., v. 35, p. 802-808, 1990.

SCHUTYSER, M. A. I.; BRIELS, W. J.; BOOM, R. M.; RINZEMA, A. Combined discrete particle and continuum model predicting solid-state fermentation in a drum fermenter. Biotechnol Bioeng., v. 86, p. 405-413, 2004.

THOMÉO, J. C.; ROUILLER, C. O.; FREIRE, J. T. Experimental analysis of heat transfer in packed beds with air flow. Ind. Eng. Chem. Res., v. 43, p. 4140-4148, 2004.

VON MEIEN, O.F.; MITCHELL, D.A. A two-phase model for water and heat transfer within an intermittently-mixed solid-state fermentation bioreactor with forced aeration. Biotechnol. Bioeng., v.79, p.416-428, 2002.

VORTMEYER, D.; HAIDEGGER, E. Discrimination of three approaches to evaluate heat fluxes for wall-cooled fixed bed chemical reactors. Chem. Eng. Sci., v. 46, p. 2651-2660, 1991.

WHITAKER, S. Forced convection heat transfer correlations for flow in pipes, past flat plates, single cylinders, single spheres, and for flow in packed beds and tube bundles. AIChE J., v. 18, p. 361-371, 1972.

ZANElATO, A. I.; SHIOTA, V. M.; GOMES, E.; DA SILVA, R.; THOMÉO, J. C. Endoglucanase production with the newly isolated Myceliophthora sp. I-1D3b in a packed bed solid state fermentor. Braz. J. Microbiol., v. 43, p. 1536-1544, 2012. 